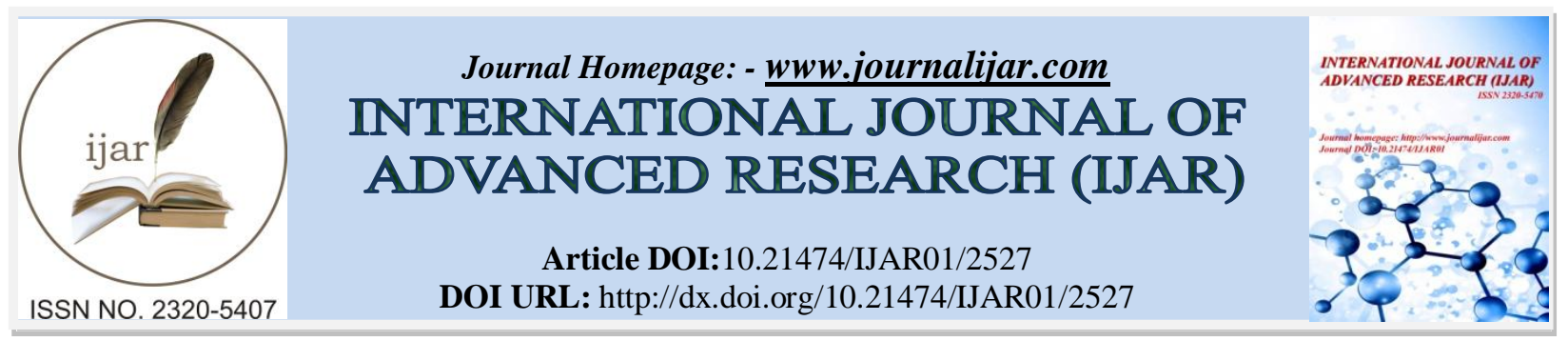

REVIEW ARTICLE

\title{
A SURVEY ON ATM FOR VISUALLY CHALLENGED PEOPLE.
}

\section{Aneesh Chandran ${ }^{1}$, Christy Robert ${ }^{2}$ and Dhanya P Iyer ${ }^{2}$.}

1. Assistant Professor, Department of CSE,Jyothi Engineering College, India.

2. B. tech Student,Department of CSE, Jyothi Engineering College, India.

\section{Manuscript Info}

\section{Manuscript History}

Received: 25 October 2016

Final Accepted: 23 November 2016

Published: December 2016

Key words: -

ATM, visually challenged, fingerprint authentication, voice commands,Blind Mode.

\section{Abstract}

Surveys says that about 314 million people are visually challenged worldwide. This paper reviews various methods used for implementing easily accessible and secure Automated Teller Machine (ATM) systems for visually challenged people. And our project is to setup an ATM for visually challenged people. The main features included is fingerprint authentication and transactions based on voice commands. The system strictly permits single person at a time inside the cabin. Door will be locked when the sensors detect human presence inside the cabin. There will be two modes of transactions Normal Mode and Blind Mode. Normal Mode is same as that of traditional ATM. Special Mode is designed for visually challenged people. A customer which is verified by fingerprint authentication can select their respective modes through voice command. In both modes, there is a provision to choose whether to check balance or to withdraw cash. In Blind Mode all the operations will be done through voice commands. Speaker system and a number of buzzers are attached to safely direct the blind person inside the cabin.

Copy Right, IJAR, 2016, All rights reserved.

\section{Introduction: -}

About 285 million people are visually impaired and about 35 million are blind worldwide. One of the problems they face in their daily life is during ATM transactions. The Automated Teller Machine (ATM) is a machine which facilitates basic banking services such as, withdrawal of money, deposition of money and checking of one's own balances etc. ATM does most of the functions of cashier in the bank. Though ATMs are inscribed with Braille for blind people, it does not completely eradicate the problems faced by blind people. Because all the blind people need not know Braille.

There also exists possibility of misusing their authentication details by the people assisting them during transactions. One of the existing solution for these problems are Talking ATMs. But still exists the possibility of leaking the authentication details.

An optimal solution for eliminating these problems is to apply fingerprint authentication. Our system ensures a two level security for the user. When the user reaches the counter the sensors attached detect the presence of human. After entering the cabin, the door is locked to confirm the security of transaction. Thereafter he can insert the smart card to the card slot and also place the finger to fingerprint slot. Fingerprint encryption is done using RSA algorithm. Once the user is verified, he can proceed to transactions. Commands for the transaction is given through voice 
commands. A number of buzzers are attached inside the cabin to direct the user to reach the machine, to place the finger, to collect the cash etc. Speakers fixed to the PC also supports the blind for a safe and secured transaction.

\section{Fingerprint Authentication: -}

Authentication is that process through which the service confirms the claim of a person to use a particular identity by the use of credentials. Biometrics is authentications based on some physical characteristics of the human body. There are two types of biometric identifiers, include physiological and behavioral characteristics. Physiological characteristics are related to the pattern of the body such as: fingerprint recognition, face recognition, DNA identification, palm print, hand geometry and iris recognition. Behavioral characteristics are related to the behavior of a person, typing rhythm, gait, digital signature and voice. We adopted the usage of fingerprint to authenticate a user since fingerprint is unique and also it is cheap to setup.

Fingerprint processing consists of two phases: fingerprint enrollment and fingerprint matching. While enrolling, user needs to enter the finger two times. The system, after processing the two-time fingerprint images, will generate a template of the fingerprint based on the processing results and stores the template. When matching, user enters the finger through optical sensor and system generates a template of the fingerprint and correlate it with templates in the finger library.

\section{RSA Algorithm: -}

RSA is an asymmetric cryptographic algorithm which is based on the complexity in factorization of large integers. RSA is the abbreviation of Ron Rivest, Adi Shamir and Leonard Adleman, who first publicly portrayed the algorithm. A user of RSA creates and then publishes the product of two large prime numbers, along with an auxiliary value, as their public key. The prime factors have to be kept secretly. Anyone can use the public key to encrypt a message. The RSA algorithm involves three steps: key generation, encryption and decryption. In our system, RSA algorithm can be used for the encryption of the fingerprint template which provides a higher level of security from intruder attacks.

\section{Voice Control: -}

Voice-controlled automatic teller machines were devised to assist people with visual impairments, inclusive of some elder people to facilitate financial transactions. Not every blind person can read Braille, and so ATM's equipped with Braille keypads don't always suffice. In this scenario user speaks into the microphone. microphone capture sound waves and digital signal signals generated. Text To Speech(TTS) converters also helps for better performance.

\section{Literature Survey: -}

Paper [1] describes an approach wherein both the username and PIN for the ATM machine can be input using British Sign Language. It elaborates a cost effective setup and also a high speed algorithm for hand gesture recognition. All algorithms were first designed and developed in MATLAB 2011b and then later deployed as software using the Java programming language.

In paper [2] an ATM processing system is implemented to illustrate the possibility of using simple hand gesture as input which provides security. The efficiency of the system is based on how well the system understand the change in hand gestures. The System is helpful not only for visually disabled people but even illiterate people can use the system and blind need not learn Braille as well. All that they need to know is the Indian sign language of digits.

Paper [3] presented a prototype layout of an ATM access system which uses finger print technology. The system architecture includes finger print module, DC motor, LCD display. And all are interfaced to the PIC microcontroller. When a user places his fingerprint to the finger print module, the micro controller compares it with user database and displays the relevant details on the LCD display. When an authorized person is verified using finger print module the door is accessed using DC motor attached. 
The paper [4] analyzes a high level model for the modification of traditional ATM systems to economically consolidate fingerprint authentication system. The paper come up with a framework for customer identification and authentication in Automated Teller Machines using Personal Identification Numbers (PIN), fingerprints and magnetic stripe cards as opposed to the traditional PIN and magnetic stripe cards verification method.

In the paper [5], author proposed a fingerprint-matching approach, which is depend on standardized fingerprint model to manufacture fingerprint from original templates. From the fingerprint templates in the database, we select one as mean images and apply Genetic Algorithms to find the conversions among them. Then, according to these transformations fingerprints are synthesized. Finally, a matching is done to show the effectiveness of the model.

The paper [6] conducts a comparative study between two algorithms for fingerprint matching. Two algorithms were compared with a common database using MATLAB. Perhaps the most important fact understood through this paper is that the most efficient and effective method to improve the verification for any given system is to combineknown algorithms in a way that we can capitalize on the advantages of each and use them to overcome the shortcomings of the complementing techniques.

In paper [7] author proposed a method which is capable for the identification of the persons in a more accurate manner. Miniature and PHOG features were extracted based on the different feature extraction methods. The extracted features were encrypted in-order to keep it more secure. The encryption and decryption process is done using RSA algorithm. The classification method used increases the accuracy of the process since the classifier employs fuzzy logic and distance metric for the classification process.

In paper [8] author tried to scheme out an authenticated way of securing our speech message. Speech message is converted into text. Then this secret text is fed to an encryption technique very similar to the RSA encryption. Here encryption is done using a unique set of keys that can only be generated by unique individual using his/her fingerprint. Moreover, the two set of keys increment the security level and hence protects our invaluable data from third unwanted parties

In the paper [9] author discuss about a unique method to ensure privacy by merging two different fingerprint patterns into a new one. The patterns of two different fingers are extracted and the minutiae positions of first pattern are overlapped with the orientation of the next one. The new template thus obtained is set as the virtual identity. On applying RSA algorithm to this virtual identity; a PKI key is obtained. These keys are in-turn used to store information in the database

The paper [10] proposes a novel method called Biometric-Crypto system for sending voice over internet. A secret key is generated from the fingerprint and is used for encryption and decryption of voice data packets. The key generation process is performed by powerful RSA algorithm. It is using this secret key that the voice data packet is encrypted as well as decrypted. It ensures better security

Paper [11] suggests a new method for authentication i.e. iris recognition. As iris pattern is unique it provides a secure method for authentication. Once the authentication phase is completed, any further transaction is performed using voice commanding by giving instructions through microphone. Iris recognition is performed in three steps namely; pre-processing, feature extraction and matching. This system not only promises security but also provides an opportunity to the differently abled ones.

Paper [12] suggests on implementing a innovative system which assists the visually impaired people in accessing email. Information is exchanged via speech format. The speech format is converted into text and is sent to the receiver. The text input is then converted to speech at the receiving end. Blind user can access mail via voice commands. Some predefined keywords like read, compose, send etc. are used.

The paper [13] puts forward a new system where the access is authorized by just speaking through a microphone which is attached to the machine. It ensures that only authorized person is accepted whereas the unauthorized person is rejected. There are two phases mentioned in the system one being testing phase and the next being the training phase. Speaker recognition is performed in two steps which text dependent and other is being text independent.

The paper [14] proposes the concept of the voice controlled Robert which can be controlled through specific voice commands. The voice module attached within (Easy V R), processes the input speech received. As soon as the input 
is received, the command is passed to the microcontroller of the robot which in turn analyses the message so as to perform necessary actions. The input speech signals are converted into digital signals by the module. These signals are transmitted through ZigBee module to the robot.

The main objective described in paper [15] is to develop an embedded system which can be employed for ATM security. In this method, during the registration phase in bank; the bankers collect the fingerprint sample as well as the mobile number of the user. The details are then stored in the database. When the user accesses the ATM, he places his finger on the module. This fresh sample is compared with the one stored in the database; if the sample is matched, then a four-digit pin is sent to the enrolled phone number which is used as the OTP for transaction.

The traditional methods of ATM authentications have many security issues, so paper [16] employs a method of employing fingerprints as unique passwords. The fingerprint sensor identifies and stores the pattern in the form of a template. When the user accesses the ATM and enters the fingerprint; matching operation is performed. If authenticated, transactions can be performed. Else, two more chances are given to the user. If the patterns remain unmatched still; then buzzer will get switched on and an alarming message is sent to the original user's mobile number.

\section{Conclusion: -}

An automated teller machine (ATM) is an electronic banking channel, which grants customers to complete basic transactions without the assistance of a branchrepresentative. This paper reviews about the accessibility of visually disabled people towards ATM. Fingerprint authentication, voice controlled transactions, encryption using RSA algorithm etc. are some of the technology which helps in designing a secure ATM for blind people. This paper conducts a detailed study on these technologies. Various types of biometric authentication systems were analyzed.

\section{References: -}

1. Sudhir Rao Rupanagudi, Ajani B. S., Varsha G. Bhat, K. Surabhi, P.R. $\quad$ Reshma, Shruthi $\quad$ G, Sarayu K. P, Sangeetha R, Rajesh Rao B, Vasanti S, "A High Speed Algorithm for Identifying Hand Gestures for an ATM Input System for the Blind", 2015 IEEE Bombay Section Symposium (IBSS).

2. Shruthi.G, Sarayu.K.P, Sangeetha.R1, Sanjoy Das, "Design of ATM Accessing System for Blind using RealTime Video Processing through Gestures", International Journal of Computer Applications (0975 - 8887) Volume 119 - No.11, June 2015.

3. DhirajSunehra, "Fingerprint Based Biometric ATM Authentication System", International Journal of Engineering Inventions, e-ISSN: 2278-7461, p-ISSN: 2319-6491, Volume 3, Issue 11 (June 2014) PP: 22-28.

4. H. Lasisi, A.A. Ajisafe, "Development of Stripe Biometric Based Fingerprint Authentications System in Automated Teller Machines", 2012 2nd International Conference on Advances in Computational Tools for Engineering Applications

5. Le Hoang Thai, Ha Nhat Tam, "Fingerprint recognition using standardized fingerprint model", IJCSI International Journal of Computer Science Issues, Vol. 7, Issue 3, No 7, May 2010, ISSN (Online): 1694-0784, ISSN (Print): 1694-0814.

6. Mary LourdeR,Dushyant Khosla, "Fingerprint Identification in Biometric Security Systems", International Journal of Computer and Electrical Engineering, Vol. 2, No. 5, October 2010,1793-8163

7. M. Malarvizhi M. Madlin Asha S. Sinduja, "Finger Print Matching based on Miniature and PHOG Feature Extraction", International Journal of Advanced Research in Computer Science and Software Engineering, Research Paper, Volume 5, Issue 10, October-2015 ISSN: 2277 128X.

8. Sayani Chandra, Sayan Paul, BidyutmalaSaha, SourishMitra, "Generate an Encryption Key by using Biometric Cryptosystems to secure transferring of Data over a Network", IOSR Journal of Computer Engineering (IOSRJCE) e-ISSN: 2278-0661, p- ISSN: 2278-8727Volume 12, Issue 1 (May. - Jun. 2013), PP 16-22.

9. Safnitha P Y, Sheena Kurian K, "Enhancing Security with Fingerprint Combination Using RSA Algorithm", International Journal of Advanced Trends in Computer Science and Engineering, Vol.3, No.4, Pages: 61-65 (2014).

10. Mrs.R.Sridevi , S.Karthika, "Biometric Cryptosystem for VoIP Security using RSA Key Generation", International Journal of Software and Web Sciences, 7(1), December 2013- Feburery 2014, pp. 25-29.

11. R.D.Salagar, AkshataPatil, "Voice Enabled ATM Machine With Iris Recognition for Authentication", Proceedings of 3rd IRF International Conference, 10th May-2014, Goa, India, ISBN: 978-93-84209-15-5. 
12. Mrs.K.M.Sanghavi, Radhika Maru, PayalKumat, AnkitaKatariya, RuchikaDudhediya, "Communication media for Blinds Based on Voice", International Journal of Modern Trends in Engineering and Research, e-ISSN No.:2349-9745, Date: 2-4 July, 2015.

13. Yekini N.A., Itegboje A.O., Oyeyinka I.K., Akinwole A.K., "Automated Biometric Voice-Based Access Control in Automatic Teller Machine (ATM)", International Journal of Advanced Computer Science and Applications, Vol. 3, No.6, 2012.

14. K. Kanna1, Dr. J. Selvakumar, "Arduino Based Voice Controlled Robot", International Research Journal of Engineering and Technology (IRJET) e-ISSN: 2395-0056 Volume: 02 Issue: 01 | Mar-2015

15. Pennam Krishnamurthy, M. Maddhusudhan Reddy, "Implementation of ATM Security by Using Fingerprint recognition and GSM", International Journal of Electronics Communication and Computer Engineering, Volume 3, Issue (1) NCRTCST, ISSN 2249 -071X.

16. Avinash Kumar Ojha, "ATM Security using Fingerprint Recognition", International Journal of Advanced Research in Computer Science and Software Engineering, Volume 5, Issue 6, June 2015 ISSN: 2277 128X. 